\title{
Donor breast milk versus infant formula for preterm infants: systematic review and meta-analysis
}

\author{
Catherine A Boyd, Maria A Quigley, Peter Brocklehurst
}

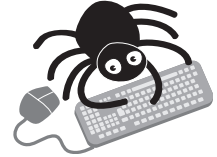

A supplementary appendix is available at hitp:// adc.bmj.com/supplemental

See end of article for authors' affiliations

......................

Correspondence to: Maria Quigley, National Perinatal Epidemiology Unit, University of Oxford, Old Road Campus, Headington, Oxford OX3 7LF, UK; maria. quigley@npeu.ox.ac.uk

Accepted 28 February 2006 Published Online First 5 April 2006
Arch Dis Child Fetal Neonatal Ed 2007;92:F169-F175. doi: 10.1136/adc.2005.089490

Objectives: To compare the effect of donor breast milk with infant formula in preterm infants. Separate comparisons with formula were made for donor breast milk that was: (1) given as a sole diet; (2) given as a supplement to mother's own breast milk; and (3) fortified with macronutrients and micronutrients. The main outcomes were death, necrotising enterocolitis (NEC), infection, growth and development.

Data sources: Electronic databases - Cochrane, CENTRAL, MEDLINE, EMBASE, CINAHL, and HMIC: DH.

Review methods: Systematic review and meta-analysis of trials and observational studies of preterm or low birthweight infants.

Results: Seven studies (including five randomised controlled trials), all from the 1970s and 1980s, fulfilled the inclusion criteria. All studies compared the effect of sole donor breast milk with formula (combined $n=471$ ). One of these also compared the effect of donor breast milk with formula given as a supplement to mother's own milk $(n=343)$. No studies examined fortified donor breast milk. A meta-analysis based on three studies found a lower risk of NEC in infants receiving donor breast milk compared with formula (combined RR 0.21, $95 \% \mathrm{Cl} 0.06$ to 0.76 ). Donor breast milk was associated with slower growth in the early postnatal period, but its long-term effect is unclear.

Conclusion: Donor breast milk is associated with a lower risk of NEC and slower growth in the early postnatal period, but the quality of the evidence is limited. Further research is needed to confirm these findings and measure the effect of fortified or supplemented donor breast milk.
B reast milk is the recommended form of enteral nutrition for all infants, including those born preterm. ${ }^{1}$ Donor breast milk is an alternative form of milk when the mother's own milk is not available or is in short supply. The advantages of breast milk over infant formulas include:

- the presence of active enzymes that enhance the maturation of the underdeveloped gut $^{2}$;

- earlier tolerance of full enteral feeding;

- anti-infective properties which protect the newborn from infection.

Although the nutrient concentrations in preterm breast milk tend to be either the same as or higher than those in term breast milk, there is concern that breast milk, whether maternal or donated, may be inadequate to support the increased nutritional requirements of the preterm or very low birthweight infant. $^{45}$

The use of donor breast milk varies across the world. Currently in the UK, 17 human milk banks supplying 50-60 neonatal units (Gillian Weaver, UK Association for Milk Banking, personal communication, 2004). In these banks all donor milk is frozen following Holder pasteurisation (heated to $62.5^{\circ} \mathrm{C}$ for $30 \mathrm{~min}$ ). However, this not only inactivates HIV, cytomegalovirus and other viruses, it also affects the nutritional and immunological properties of breast milk. ${ }^{3}$ For example, it is estimated that $34 \%$ of the small amount of immunoglobulin $\mathrm{G}$ is destroyed, although most of the secretory immunoglobulin A remains $(0-30 \%$ destroyed). ${ }^{2}$ As donor milk is usually provided by women who deliver at term, and is pasteurised, it cannot be presumed that it will have the same effect as mother's own milk.

Although several systematic reviews have compared the effect of breast milk with infant formula in preterm infants, ${ }^{4-7}$ none has specifically focused on pasteurised donor breast milk or separated out the effects of donor breast milk given as a sole diet and that given as a supplement to mother's own milk. The aim of this systematic review, therefore, was to compare the effects of pasteurised donor breast milk and infant formula in preterm infants. We separated the effects of donor milk given as a sole diet, donor milk given as a supplement to mother's own milk, and donor milk that was fortified with macronutrients or micronutrients to encompass the current provision of donor milk in clinical practice. The main outcomes of interest were death, necrotising enterocolitis (NEC), infection, growth and development. We did not investigate whether pasteurisation adequately eliminates microbial contaminants.

\section{METHODS}

\section{Search strategy}

We carried out standard search strategies using the databases Cochrane, CENTRAL, MEDLINE, EMBASE, CINAHL and HMIC: DH from their inception to August 2004. Details of the search strategy can be found in the appendix (see http:// adc.bmj.com/supplemental). There was no language restriction. There was no systematic attempt to search the "grey" literature although some articles were found in the references for indexed articles.

\section{Selection}

We included studies on the basis of study design, population and comparison groups (box 1). All donor breast milk had to be donated from someone other than the infant's mother and it had to be pasteurised. All clinical outcomes were included.

Abbreviation: NEC, necrotising enterocolitis 


\section{Box 1 Inclusion criteria}

Study design

- Randomised controlled trials (RCTs), quasi-RCTs and observational studies.

Population

- Preterm (<37 weeks' gestation) or low birthweight $(<2500 \mathrm{~g})$ infants.

Types of intervention/exposure

- Sole diet of pasteurised donor milk $v$ sole diet of formula.

- Mother's own milk supplemented with pasteurised donor milk $v$ mother's own milk supplemented with formula.

- Fortified, pasteurised donor milk $v$ fortified formula.

Outcome measures

- All clinical outcomes were considered. The primary outcomes were necrotising enterocolitis and infection. All other short-term and long-term health and development outcomes including any data on adverse events were considered as secondary outcomes.

- Studies which only assessed the risk of transmissible diseases from milk banks were excluded.

\section{Data abstraction}

The articles identified by our search strategy were screened (title and abstract) by two independent reviewers (CB and $\mathrm{MQ})$. Then those articles which potentially met the inclusion criteria were critically reviewed. The decision to include or exclude a specific article was made by consensus of the two reviewers. For two studies, information on whether the milk was pasteurised was not available in the published reports but was supplied by the authors of the primary studies.

\section{Validity assessment}

We assessed all studies for methodological quality in terms of sample size estimation, proportion followed up, blinding of outcome assessment, comparison of baseline variables and assessment of confounding. The RCTs were also assessed for method of randomisation, allocation concealment and blinding of intervention.

\section{Quantitative data synthesis}

Data were extracted and summarised into evidence tables, which included effect measures with 95\% CIs. Where outcome measures were given in different units across studies, these were made consistent where possible. Meta-analyses were planned if there were sufficient data, and it was anticipated that a fixed-effects model would be used unless there was evidence of significant heterogeneity $(\mathrm{p}<0.10)$. We conducted and reported this review in accordance with the guidelines set out in the Quality Of Reporting Of Meta-analyses (QUOROM) statement. ${ }^{8}$

\section{RESULTS}

The search produced 1594 articles which were screened (title and abstract). Of these, 242 potentially addressed the research questions but on reading the articles only 16, arising from 7 studies, met the inclusion criteria (fig 1).

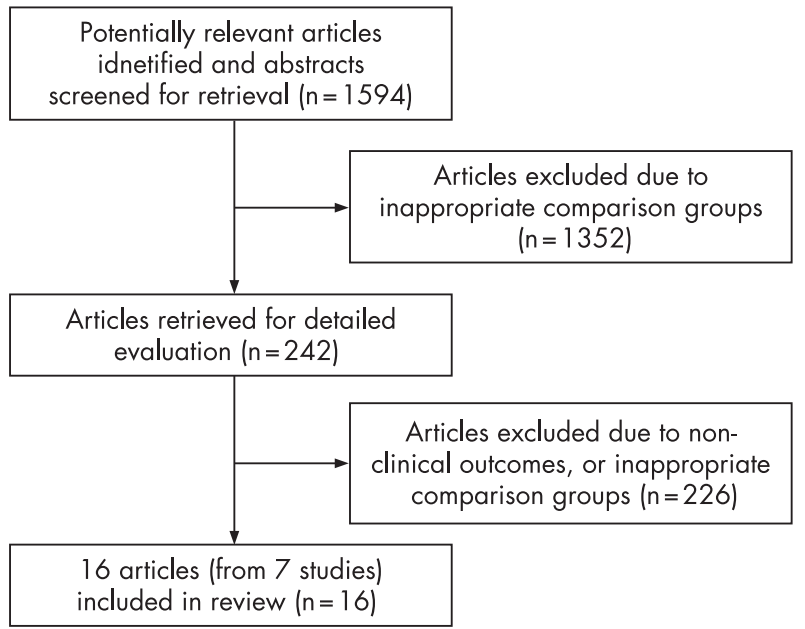

Figure 1 Results of search and selection of articles (QUOROM statement flow diagram ${ }^{8}$ )

\section{Study characteristics}

Table 1 provides details of the characteristics of the seven included studies. ${ }^{9-24}$ The combined sample size of the studies which compared the sole diet of donor breast milk with sole formula was 471 infants. Three of these studies started in the 1970s (in Wales, ${ }^{9}$ Finland $^{10}$ and Hungary ${ }^{11}$ ) and four in the 1980s (in the USA, ${ }^{12}$ England, ${ }^{13-22}$ South Africa ${ }^{23}$ and France ${ }^{24}$ ). One of these studies ${ }^{13-22}$ (Lucas et al $^{13}$ will be cited as the primary paper for this study) also compared donor milk with formula, both given as a supplement to mother's own milk. This study was conducted in England in the 1980s, with a sample size of $343 .{ }^{13}$ We did not find any other studies comparing donor milk with formula as a supplement to mother's own milk. No studies were found comparing fortified donor milk with formula.

There was considerable variability between the studies in terms of the type of donor milk (preterm $v$ term; drip $v$ expressed) and formulas used, the feeding regimens and the period of intervention. The study population also varied in terms of mean gestation (range 30.2-33.2 weeks) and mean birth weight (range 1310-1954 g) (table 1).

\section{Methodological quality of studies}

The methodological quality of the studies is summarised in table 2. Five studies were RCTs, however, only one of these trials ${ }^{13}$ reported that a sample size estimate had been calculated prior to the trial starting. This study also had adequate methodological quality in terms of allocation concealment and randomisation. None of the remaining four RCTs included a sample size estimate, and two did not specify the method of randomisation. ${ }^{911}$ One of the studies did not specify whether it was randomised ${ }^{24}$ One study, ${ }^{23}$ which was designed as a trial, stopped recruitment in the formula group due to an "outbreak" of NEC, and continued to recruit in the formula group from another hospital. We have only used outcome data from the first hospital.

\section{Findings of the included studies}

Tables 3-5 present the main results of the seven studies included in the review.

\section{Effect on mortality}

Mortality was reported in one study ${ }^{13}$ for both sole diet and supplementary diet comparisons. This study found a lower risk of mortality at ages 9 months and 18 months in the donor milk group compared with the formula group, for both sole and 
Table 2 Methodological quality of the studies included in the review

\begin{tabular}{|c|c|c|c|c|c|c|c|c|}
\hline Study & $\begin{array}{l}\text { Sample size } \\
\text { estimate }\end{array}$ & Method of randomisation & $\begin{array}{l}\text { Allocation } \\
\text { Concealment }\end{array}$ & $\begin{array}{l}\text { Blinding of } \\
\text { intervention }\end{array}$ & $\begin{array}{l}\text { Proportion }(\%) \text { included } \\
\text { in analysis* }\end{array}$ & $\begin{array}{l}\text { Blinding of } \\
\text { outcome }\end{array}$ & $\begin{array}{l}\text { Baseline variables } \\
\text { compared }\end{array}$ & Assessment of confounding \\
\hline Davies $^{9}$ & No & $\begin{array}{l}\text { Randomised but method not } \\
\text { stated }\end{array}$ & Not stated & Not stated & $100 \%$ & Not stated & Yes & Stratification by gestational age \\
\hline Raiha et $a l^{10}$ & No & Blocked randomisation & Coding of bottles & Not stated & $95 \%$ & Not stated & Yes & Stratification by gestational age \\
\hline Schultz et al" & No & $\begin{array}{l}\text { Randomised but method not } \\
\text { stated }\end{array}$ & Not stated & Not stated & $100 \%$ & Not stated & Yes & No \\
\hline Gross $^{12}$ & No & Random number table & Not stated & Not stated & $100 \%$ & Not stated & Yes & No \\
\hline Lucas et $a l^{13-22}$ & Yes & Blocked randomisation & $\begin{array}{l}\text { Opaque, sealed, } \\
\text { numbered envelopes }\end{array}$ & No & $\begin{array}{l}100 \% \text { for short term } \\
\text { outcomes } 69 \% \text { at } 9 \text { months } \\
86 \% \text { at } 18 \text { months } \\
82 \% \text { at } 8 \text { years }\end{array}$ & For some outcomes & Yes & For some outcomes \\
\hline Cooper ef $a f^{33}$ & No & $\begin{array}{l}\text { Not random (alternating } \\
\text { assignment) }\end{array}$ & Not stated & Not stated & $100 \%$ & Not stated & Yes & No \\
\hline Putet ef $a l^{24}$ & No & $\begin{array}{l}\text { Not stated (may not be } \\
\text { randomised) }\end{array}$ & Not stated & Not stated & $92 \%$ & Not stated & Yes & No \\
\hline
\end{tabular}

supplementary diet comparisons, but this effect was not statistically significant (table 3 )

\section{Effect on NEC}

In the sole diet comparison, NEC was reported in three studies. ${ }^{12}{ }^{1323}$ In all of these studies, there was a lower risk of NEC in the donor milk group than the formula group but the effect was not statistically significant (table 3). For confirmed cases of NEC, the three studies yielded almost identical measures of effect (risk ratio (RR) 0.21-0.22) with similar 95\% CIs (the largest study had a much lower overall risk of NEC and hence had a similar level of precision to the smallest study). In view of the homogeneity of these RRs, a fixed-effects meta-analysis and a random-effects meta-analysis gave identical results (combined RR $0.21,95 \%$ CI 0.06 to $0.76, \mathrm{p}=0.017$ ). The combined evidence from these studies suggests that donor milk reduces the risk of NEC by about $79 \%$ (95\% CI $24 \%$ to 94\%) (fig 2).

The risk of confirmed NEC in the formula group in these three studies was 5.3\%, ${ }^{13} 11.5 \%{ }^{12}$ and $20.0 \%{ }^{23}$ Each study yielded different estimates of the risk difference (RD) $4.1 \%$, $95 \%$ CI $-1.4 \%$ to $9.6 \%)^{13} ; 9.2 \%(95 \% \text { CI }-4.1 \% \text { to } 22.3 \%)^{12}$; $15.5 \%$ ( $95 \%$ CI $-5.9 \%$ to $37.6 \%{ }^{23}$ ). The combined estimate of the $\mathrm{RD}$, estimated from a random effects meta-analysis, was $5.4 \%$
(95\% CI $0.5 \%$ to $10.3 \%, p=0.032$ ). This suggests that in settings in which the risk of NEC in the formula group is about $5-20 \%$, approximately 18.5 preterm infants ( $95 \%$ CI 9.7 to 200) would need to be given donor milk to prevent one case of NEC.

In the supplementary diet comparison, ${ }^{13}$ there were more suspected cases of NEC but fewer confirmed cases in the donor milk group than in the formula group but neither effect reached statistically significance (table 3 ).

\section{Effect on other perinatal outcomes}

Four studies in the sole diet comparison reported other perinatal morbidity events. Three studies found significantly fewer episodes of feeding intolerance (including NEC) and diarrhoea in the donor milk group compared with the formula group (table 3); in one, ${ }^{13}$ infants in the donor milk group were found to tolerate full enteral feeds earlier, another study ${ }^{12}$ reported significantly fewer withdrawals due to feeding intolerance (within 9-31 days of starting the intervention) in the donor milk group, and yet another ${ }^{11}$ found infants fed donor milk had significantly fewer episodes of mild diarrhoea in the first two weeks after birth. One study ${ }^{23}$ reported more withdrawals due to respiratory symptoms within the first few days of starting the intervention in the donor milk group compared with the formula group $(12.5 \% \vee 6.7 \%)$, but this

Table 3 Comparisons of mortality, NEC, and other perinatal outcomes between the donor breast milk and formula groups

\begin{tabular}{|c|c|c|c|c|c|c|c|c|c|c|c|c|}
\hline \multirow[b]{3}{*}{ Outcome and studies } & \multicolumn{6}{|c|}{ Sole diet comparison } & \multicolumn{6}{|c|}{ Supplementary diet comparison } \\
\hline & \multicolumn{2}{|c|}{ Donor breast milk } & \multicolumn{2}{|c|}{ Formula } & \multirow[b]{2}{*}{$\operatorname{RR}(95 \% \mathrm{Cl})$} & \multirow[b]{2}{*}{ p value } & \multicolumn{2}{|c|}{ Donor breast milk } & \multicolumn{2}{|c|}{ Formula } & \multirow[b]{2}{*}{$\operatorname{RR}(95 \% \mathrm{Cl})$} & \multirow[b]{2}{*}{$p$ value } \\
\hline & No. & $n(\%)$ & No. & $n(\%)$ & & & No. & $n(\%)$ & No. & $n(\%)$ & & \\
\hline \multicolumn{13}{|c|}{ Mortality up to 9 months } \\
\hline Lucas et al ${ }^{17}$ & 83 & $7(8.4)$ & 76 & $9(11.8)$ & 0.71 (0.28 to 1.82$)$ & $p=0.48$ & 170 & $12(7.1)$ & 173 & $15(8.7)$ & $0.81(0.39$ to 1.69$)$ & $p=0.58$ \\
\hline $\begin{array}{l}\text { Mortality up to } 18 \mathrm{mo} \\
\text { Lucas ef } \mathrm{al}^{71}{ }^{19}\end{array}$ & 83 & $7(8.4)$ & 76 & $9(11.8)$ & 0.71 (0.28 to 1.82$)$ & $p=0.48$ & 170 & $13(7.6)$ & 173 & $16(9.2)$ & $0.83(0.41$ to 1.67$)$ & $p=0.59$ \\
\hline \multicolumn{13}{|l|}{ Confirmed NEC } \\
\hline Cooper et $a f^{3}$ & 24 & $1(4.2)$ & 15 & $3(20.0)$ & 0.21 (0.02 to 1.82$)$ & $p=0.113$ & & & & & & \\
\hline Gross $^{12}$ & 41 & $1(2.4)$ & 26 & $3(11.5)$ & 0.21 (0.02 to 1.92$)$ & $p=0.126$ & & & & & & \\
\hline Lucas and Cole ${ }^{18}$ & 86 & $1(1.2)$ & 76 & $4(5.3)$ & 0.22 (0.03 to 1.93$)$ & $p=0.13$ & 167 & $2(1.2)$ & 173 & $5(2.9)$ & $0.41(0.08$ to 2.11$)$ & $p=0.27$ \\
\hline \multicolumn{13}{|l|}{ Suspected NEC } \\
\hline Lucas and Cole ${ }^{18}$ & 86 & $3(3.5)$ & 76 & $6(7.9)$ & $0.44(0.11$ to 1.71$)$ & $p=0.22$ & 167 & $8(4.8)$ & 173 & $6(3.5)$ & $1.38(0.49$ to 3.90$)$ & $p=0.54$ \\
\hline \multicolumn{13}{|l|}{ Respiratory symptoms } \\
\hline Cooper et a P $^{3}$ & 24 & $3(12.5)$ & 15 & $1(6.7)$ & $1.87(0.21$ to 16.41$)$ & $p=0.56$ & & & & & & \\
\hline \multicolumn{13}{|c|}{ Mild diarrhoea (culture negative) } \\
\hline Schultz et al ${ }^{11}$ & 10 & $0(0)$ & 10 & $6(60)$ & 0 & $p=0.011$ & & & & & & \\
\hline \multicolumn{13}{|c|}{ Feeding intolerance, including NEC } \\
\hline Gross $^{12}$ & 41 & $1(2.4)$ & 26 & $6(23.1)$ & 0.11 (0.01 to 0.83$)$ & $p=0.007$ & & & & & & \\
\hline \multicolumn{13}{|c|}{$>2$ weeks to tolerate full enteral feeds } \\
\hline Lucas $^{16}$ & 83 & $9(11)$ & 76 & $25(33)$ & $0.33(0.16$ to 0.66$)$ & $p=0.0007$ & & & & & & \\
\hline \multicolumn{13}{|c|}{$>3$ weeks to tolerate full enteral feeds } \\
\hline Lucas $^{16}$ & 83 & $4(5)$ & 76 & $13(17)$ & 0.28 (0.10 to 0.83$)$ & $p=0.012$ & & & & & & \\
\hline
\end{tabular}

NEC, necrotising ulcerative colitis; No., total; $n$, number of cases.

$\mathrm{RR}<1$ favours donor breast milk. 


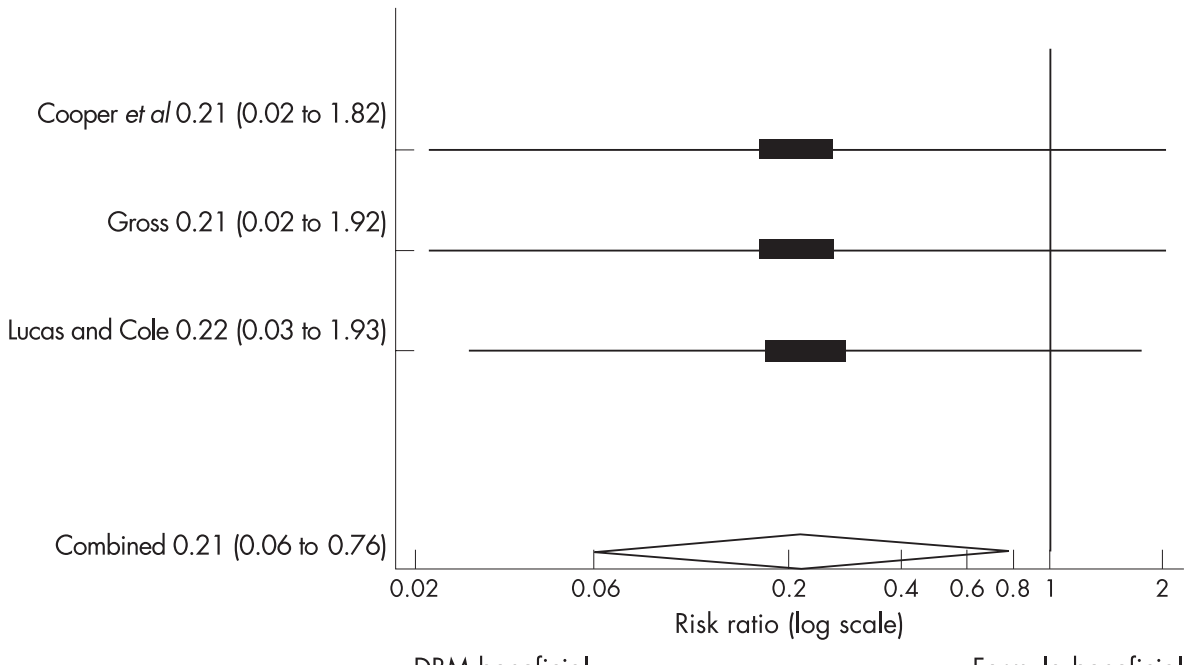

DBM beneficial
Figure 2 Individual and combined estimates of risk ratio of confirmed NEC for sole DBM versus sole formula milk. hip ratio. Importantly, this study found significantly slower growth in the donor milk group compared with the formula group during the early postnatal period.

Similarly, in the supplementary diet comparison, the slower growth in the donor milk group compared with the formula group during the early postnatal period had no long-term effect on growth in this study; the two groups were broadly similar with the exception of body mass index being significantly higher in the donor milk group at age 18 months.

\section{Effect on developmental status}

Child development at age 9 months and 18 months was assessed in one study ${ }^{13}$ (table 5). In the sole diet comparison there were fewer neurologically impaired children at age 9 months in the donor milk group compared with the formula group but this effect was not statistically significant $(R R=0.38$, $95 \%$ CI 0.12 to 1.16 ), and was even weaker when assessed at age 18 months. There were no significant differences between the two feeding groups with respect to mean developmental quotient at age 9 months (Knobloch index) or 18 months (Bayley index) (table 5).

In the supplementary diet comparison, the proportion of neurologically impaired children at age 9 months and 18 months was similar in the donor milk group compared with the formula group. The donor milk group had significantly lower mean developmental scores than the formula group at age 9 months (Knobloch) but not when assessed at age 18 months (Bayley) (table 5).

\section{Effect on later outcomes}

One study measured systolic and diastolic blood pressure at age 7.5-8 years and found no significant differences between the donor milk and formula groups in both the sole and supplementary comparisons (see appendix table $\mathrm{F}$ at http:// adc.bmj.com/supplemental). ${ }^{20}$

\section{DISCUSSION}

Before discussing the results of this review, a number of potential limitations should be considered. First, only seven studies were included and the total sample size for most outcomes was small. Second, these studies started over 20 years ago, and they may no longer be clinically relevant to contemporary practice as survival of preterm and low birthweight infants has greatly improved ${ }^{25}$ and feeding practices have changed. Third, the methodological quality of most of the studies was poor in terms of randomisation, blinding of carers and assessors, and assessment of confounding which may have introduced bias, in particular, for the observational data included. Lastly, substantial heterogeneity between the studies makes it difficult to pool evidence across studies. The studies had different inclusion criteria and varied in terms of the type of donor and formula milk used, and the method, timing, and volume of feeding (table 1). These differences may account for some of the observed differences in effects between the studies.

Since the search was conducted, another trial has been published. ${ }^{26}$ This trial, in extremely preterm babies ( $<30$ weeks' gestation), compared fortified donor breast milk with formula, both given as a supplement to mother's own milk. The trial found no significant difference between the two groups in terms of infection-related events or death. For NEC, there was some protection, but the $95 \%$ CI was wide (RR 0.56, 0.20 to 1.58). With regard to growth, the trial revealed no effect on length or head circumference gain, but found significantly poorer weight gain in the donor milk group. This resulted in $21 \%$ of the infants randomised to donor milk being given

\section{What is already known on this topic}

- Breast milk is the recommended form of enteral nutrition for all infants.

- Pasteurised donor breast milk is an alternative when mother's own milk is not available.

- Pasteurisation affects the nutritional and immunological properties of breast milk.

- The evidence for the benefits of donor milk compared with formula for preterm infants is limited.

\section{What this study adds}

- Donor milk given as a sole diet appears to be associated with a lower risk of NEC but slower growth in the early postnatal period when compared with infant formula.

- The long-term effect of donor milk as compared with formula is unclear. 
formula; this may have diluted the effect of donor milk on outcomes such as NEC.

Our findings, based on 13 cases of NEC in 268 infants, suggest that donor milk given as a sole diet is associated with a lower risk of NEC compared with formula. Although the observed effects were remarkably similar between the three studies (RR 0.21-0.22), true heterogeneity of effect cannot be ruled out because of differences between the studies in terms of the incidence of NEC (5-20\%), the type of donor milk and the timing of feeding initiation. Furthermore, one study $y^{23}$ was not randomised, and none of the studies considered blinding of the intervention or outcome. These methodological weaknesses may have biased the observed effects, particularly in the study $y^{23}$ which did not state how NEC was diagnosed; bias due to subjective diagnosis cannot be ruled out. The homogeneous effects found in the three studies reduces the likelihood of a chance finding, and these alternative explanations are unlikely to account wholly for the large effects observed. Moreover, in the study which looked at both sole and supplemented diets, ${ }^{13}$ the risk of NEC increased as the amount of formula increased from $1.2 \%(2 / 167)$ in infants who received donor milk and mother's own milk and $1.2 \%(1 / 86)$ in infants who received donor milk only to $2.9 \%(5 / 173)$ in infants who received formula and mother's own milk and to $5.3 \%(4 / 76)$ in infants who received formula only $(p=0.053$ for linear trend). The effect of donor milk on NEC seemed weaker in the study in which donor milk and formula were given as a supplement to mother's own milk (RR $0.41,95 \%$ CI 0.08 to 2.11 ), although the study may not have had sufficient power to detect a modest effect for such a rare outcome (incidence of NEC in formula group was $2.9 \%$ ). In Schanler and colleagues' recent trial, ${ }^{26}$ there was a similar suggestion of some protection of fortified donor milk given as a supplement to mother's own milk.

Breast milk is known to have immune properties as it includes specific immunoglobulin A, lysozyme and lactoferrin. ${ }^{2}$ Therefore breast milk is thought to protect the preterm infant from bacterial and viral infections. Holder pasteurisation, however, reduces some of the anti-infective properties of breast milk. Infection was not reported as an outcome in any of the studies included in this review, but it was an outcome in the recent Schanler trial, ${ }^{26}$ although no effect was observed.

The nutrient concentrations in human milk may be inadequate for preterm or very low birthweight infants, who have increased nutritional requirements. ${ }^{2}$ This may be particularly so for donor milk, as Holder pasteurisation reduces some of the nutritive properties, ${ }^{2}$ and the composition of donor milk is not the same as that received by a mother's own infant. This is especially true for drip milk, which was used in one study, ${ }^{13}$ and term breast milk, which was used in four studies.9-12 Inadequate nutrition in the early postnatal period is thought to affect growth, bone mineralisation and neurodevelopment. One trial ${ }^{12}$ excluded infants who developed feed intolerance and NEC after randomisation, which may have exaggerated the increase in short-term growth among infants randomised to formula. Although the evidence suggests that donor milk is associated with slower growth in the early postnatal period, only one study included long-term follow-up..$^{13}$ Interestingly, this study found no difference between the groups in several growth indices and neurodevelopment at ages 9 months and 18 months, and growth and blood pressure at 7.5-8 years despite observing slower growth in the donor milk group in the short term.

Donor milk given as a sole diet is associated with a lower risk of NEC but slower growth in the early postnatal period. The long-term effect of donor milk as compared with formula is unclear because only one study followed participants into childhood and adulthood. Further research is needed to confirm our findings and measure the effect of donor breast milk that is fortified or given as a supplement to mother's own milk.

\section{Authors' affiliations \\ C A Boyd, M A Quigley, P Brocklehurst, National Perinatal Epidemiology Unit, Oxford University, Oxford, UK}

Funding: This work was undertaken by the National Perinatal Epidemiology Unit which receives funding from the Department of Health. The views expressed in this publication are those of the authors and not necessarily those of the Department of Health.

Competing interests: None declared.

\section{REFERENCES}

1 McGuire W, Henderson G, Fowlie PW. Feeding the preterm infant. BMJ 2004;329:1227-30.

2 Lawrence RA, Lawrence RM. Breastfeeding. A guide for the medical profession. St Louis, Missouri: Mosby, 1999.

3 Wight NE. Donor human milk for preterm infants. J Perinatol 2001;21:249-54

4 Henderson G. Anthony MY, McGuire W. Formula milk versus term human milk for feeding preterm or low birth weight infants (Cochrane Review). In:Cochrane Library, Issue 3. Chichester, UK: John Wiley \& Sons, 2004.

5 Henderson G, Anthony MY, McGuire W. Formula milk versus preterm human milk for feeding preterm or low birth weight infants (Cochrane Review). In: Cochrane Library, Issue 3. Chichester, UK: John Wiley \& Sons, 2004

6 de Silva A, Jones PW, Spencer SA. Does human milk reduce infection rates in preterm infants? A systematic review. Arch Dis Child Fetal Neonatol Ed 2004:89:F509-13.

7 McGuire W, Anthony MY. Donor human milk versus formula for preventing necrotising enterocolitis in preterm infants: a systematic review. Arch Dis Child Fetal Neonatol Ed 2003;88:F1 1-14.

8 Moher D, Cook J, Eastwook S, et al. Improving the quality of reports of metaanalyses of randomised controlled trials: the QUOROM statement. Lancet 1999;354:1896-900.

9 Davies DP. Adequacy of expressed breast milk for early growth of preterm infants. Arch Dis Child 1977;52:296-301.

10 Raiha NC, Heinonen K, Rassin DK, et al. Milk protein quantity and quality in lowbirthweight infants: I. Metabolic responses and effects on growth. Pediatrics 1976;57:659-84

11 Schultz K, Soltesz G, Mestyan J. The metabolic consequences of human milk and formula feeding in premature infants. Acta Paediatr Scand 1980;69:647-52

12 Gross SJ. Growth and biochemical response of preterm infants fed human milk or modified infant formula. N Engl J Med 1983;308:237-41.

13 Lucas A, Gore SM, Cole TJ, et al. Multicentre trial on feeding low birthweight infants: effects of diet on early growth. Arch Dis Child 1984;59:722-30.

14 Lucas A, Mclaughlan P, Coombs RRA. Latent anaphylactic sensitisation of infants of low birth weight to cows' milk proteins. BMJ 1984;289:1254-6.

15 Lucas A, Baker BA. Breast milk jaundice in premature infants. Arch Dis Child 1986:61:1063-7.

16 Lucas A. AIDS and human milk bank closures. Lancet 1987;8541:1092-3.

17 Lucas A, Morley R, Cole TJ, et al. Early diet in preterm babies and developmental status in infancy. Arch Dis Child 1989;64:1570-8.

18 Lucas A, Cole TJ. Breast milk and neonatal necrotising enterocolitis. Lancet 1990;336:1519-23.

19 Lucas A, Morley R, Cole TJ, et al. A randomised multicentre study of human milk versus formula and later development in preterm infants. Arch Dis Child Fetal Neonatal Ed 1994;70:F141-6.

20 Lucas A, Morley R. Does early nutrition in infants born before term programme later blood pressure? BMJ 1994;309:304-8.

21 Bishop NJ, Dahlenburg SL, Fewtrell MS, et al. Early diet of preterm infants and bone mineralization at age five years. Acta Paediatr 1996;85:230-6.

22 Morley R, Lucas A. Randomized diet in the neonatal period and growth performance until 7.5-8 y of age in preterm children. Am J Clin Nutr 2000;71:822-8

23 Cooper PA, Rothberg AD, Pettifor JM, et al. I. Growth and biochemical response of premature infants fed pooled preterm milk or special formula. J Paediatr Gastroenterol Nutr 1984;3:749-54.

24 Putet G, Senterre J, Rigo J, et al. Nutrient balance, energy utilization, and composition of weight gain in very-low-birth-weight infants fed pooled human milk or a preterm formula. J Pediatr 1984;105:79-85.

25 Surman G, Newdick $H$, Johnson A. Cerebral palsy rates among low-birthweight infants fell in the 1990s. Dev Med Child Neurol 2003;45:456-62.

26 Schanler RJ, Lau C, Hurst NM, et al. Randomized trial of donor human milk versus preterm formula as substitutes for mothers' own milk in the feeding of extremely premature infants. Pediatrics 2005; 116:400-6. 